\title{
Predictive Value of Neutrophil/Lymphocyte Ratio for Efficacy of Preoperative Chemotherapy in Triple-Negative Breast Cancer
}

\author{
Yuka Asano, $\mathrm{MD}^{1}$, Shinichiro Kashiwagi, $\mathrm{MD}, \mathrm{PhD}^{1}$, Naoyoshi Onoda, $\mathrm{MD}$, $\mathrm{PhD}^{1}$, Satoru Noda, MD, $\mathrm{PhD}^{1}$, \\ Hidemi Kawajiri, MD, $\mathrm{PhD}^{1}$, Tsutomu Takashima, $\mathrm{MD}, \mathrm{PhD}^{1}$, Masahiko Ohsawa, $\mathrm{MD}, \mathrm{PhD}^{2}$, Seiichi Kitagawa, \\ $\mathrm{MD}, \mathrm{PhD}^{\mathbf{3}}$, and Kosei Hirakawa, $\mathrm{MD}, \mathbf{P h D}^{1}$ \\ ${ }^{1}$ Department of Surgical Oncology, Osaka City University Graduate School of Medicine, Osaka, Japan; ${ }^{2}$ Department of \\ Diagnostic Pathology, Osaka City University Graduate School of Medicine, Osaka, Japan; ${ }^{3}$ Department of Physiology, \\ Osaka City University Graduate School of Medicine, Osaka, Japan
}

\begin{abstract}
Background. The neutrophil/lymphocyte ratio (NLR) has been reportedly associated with prognosis in cancer patients by influencing both cancer progression and chemosensitivity. However, the correlation between NLR and the outcome of neoadjuvant chemotherapy (NAC) in breast cancer patients remains unclear.
\end{abstract}

Methods. NLR was evaluated in 177 patients with breast cancer treated with NAC with 5-fluorouracil, epirubicin, and cyclophosphamide, followed by weekly paclitaxel and subsequent curative surgery. The correlation between NLR and prognosis, including the efficacy of NAC, was evaluated retrospectively.

Results. NLR ranged from 0.5 to 10.6 . Fifty-eight patients with low NLR $(<3.0)$ had a higher pathological complete response $(\mathrm{pCR})$ rate $(p<0.001)$ and were more frequently diagnosed with ER-negative/progesterone receptor (PR)negative/HER2-negative (triple-negative) breast cancer (TNBC; $p<0.001$ ) compared with patients with high NLR $(\geq 3.0)$. Among TNBC patients who achieved pCR, disease-free survival $(p=0.006)$ and overall survival $(p<0.001)$ were significantly longer in patients with low NLR than in those with high NLR. Low NLR was

Electronic supplementary material The online version of this article (doi:10.1245/s10434-015-4934-0) contains supplementary material, which is available to authorized users.

(C) The Author(s) 2015. This article is published with open access at Springerlink.com

First Received: 1 May 2015;

Published Online: 28 October 2015

S. Kashiwagi, MD, $\mathrm{PhD}$

e-mail: spqv9ke9@view.ocn.ne.jp associated with a significantly favorable prognosis in TNBC patients who achieved pCR, according to univariate analysis $(p=0.044$, hazard ratio $=0.06)$.

Conclusions. Low NLR may indicate high efficacy and favorable outcome after NAC in patients with TNBC.

Treatment with neoadjuvant chemotherapy (NAC) increases the rate of breast-conserving surgery and reduces the risk of postoperative recurrence in patients with resectable breast cancer. ${ }^{1-4}$ Efficacy is enhanced by the combined use of anthracyclines and taxanes, ${ }^{1,5,6}$ and further improvements in therapeutic effects may be expected with the advent of molecular-targeting drugs. ${ }^{7,8}$ In addition, evaluation of the therapeutic effects of NAC can provide valuable information.

Breast cancer can be classified into the following phenotypes: estrogen receptor (ER)-positive (luminal, normal breast-like); human epidermal growth factor receptor (HER) 2-overexpressing; and basal-like, based on cDNA microarray analysis. ${ }^{9-11}$ The basal-like subtype displays characteristics of myoepithelial cells/basal cells, and often corresponds to so-called ER-negative/progesterone receptor (PR)-negative/HER2-negative (triple-negative) breast cancer (TNBC). Patients with TNBC do not benefit from endocrine or anti-HER2 therapy, and chemotherapy is the only treatment option. ${ }^{12-16}$ However, the efficacy of chemotherapy varies among patients with TNBC, and accurate methods for predicting the outcome not only help to identify the direct treatment effects, but also help to reduce adverse events caused by inappropriate treatment.

Abnormalities in white blood cell subpopulations, such as neutrophilia and lymphopenia, have been reported in tumor-bearing patients, and the neutrophil/lymphocyte 
ratio (NLR) has been found to be associated with prognosis in patients following radical surgery. ${ }^{17-21}$ Neutrophils are known to promote tumor cell proliferation, angiogenesis, and distant metastasis, while lymphocytes play a central role in the immune reaction against tumors. NLR may thus be related to both prognosis and chemosensitivity. ${ }^{22,23}$

This single-center, retrospective study aimed to evaluate NLR as a possible markers for predicting the outcome of NAC in patients with TNBC in a consecutive patient series treated with a standardized single protocol.

\section{METHODS}

\section{Patient Background}

A total of 177 patients with resectable, early-stage breast cancer diagnosed as stage IIA (T1, N1, M0 or T2, N0, M0), IIB (T2, N1, M0 or T3, N0, M0), or IIIA (T1-2, N2, M0 or T3, N1-2, M0) were treated with NAC between 2007 and 2013. Tumor stage and $\mathrm{T}$ and $\mathrm{N}$ factors were stratified based on the TNM Classification of Malignant Tumors, UICC Sixth Edition. ${ }^{24}$ Breast cancer was confirmed histologically by core needle biopsy and staged by systemic imaging studies using computed tomography (CT), ultrasonography (US), and bone scintigraphy. Breast cancer was classified into subtypes according to the immunohistochemical expression of ER, PR, HER2, and Ki67. The cutoffs for ER positivity and PR positivity were both $>0 \%$ positive tumor cells with nuclear staining. Tumors with $3+$ HER2 on immunohistochemical staining were considered to show HER2 overexpression; tumors with 2+ HER2 were further analyzed by fluorescence in situ hybridization; and those with HER2/CER17 $\geq 2.0$ were also considered to exhibit HER2 overexpression. A Ki67-labeling index $\geq 14 \%$ tumor cells with nuclear staining was determined to be positive.

All patients received a standardized protocol of NAC consisting of four courses of FEC100 $\left(500 \mathrm{mg} / \mathrm{m}^{2}\right.$ fluorouracil, $100 \mathrm{mg} / \mathrm{m}^{2}$ epirubicin, and $500 \mathrm{mg} / \mathrm{m}^{2}$ cyclophosphamide) every 3 weeks, followed by 12 courses of $80 \mathrm{mg} / \mathrm{m}^{2}$ paclitaxel administered weekly. ${ }^{25,26}$ Fortyfive patients had HER2-positive breast cancer and were additionally administered weekly $(2 \mathrm{mg} / \mathrm{kg}$ ) or tri-weekly $(6 \mathrm{mg} / \mathrm{kg})$ trastuzumab during paclitaxel treatment. ${ }^{27}$ All patients underwent chemotherapy as outpatients. Therapeutic anti-tumor effects were assessed according to the Response Evaluation Criteria in Solid Tumors (RECIST) criteria. $^{28}$ Pathological complete response (pCR) was defined as the complete disappearance of the invasive compartment of the lesion with or without intraductal components, including in the lymph nodes. Patients underwent mastectomy or breast-conserving surgery after
NAC. All patients who underwent breast-conserving surgery were administered postoperative radiotherapy to the remnant breast. Overall survival (OS) time was the period from the initiation of NAC to the time of death from any cause. Disease-free survival (DFS) was defined as freedom from all local, loco-regional, and distant recurrences. All patients were followed up by physical examination every 3 months, US every 6 months, and CT and bone scintigraphy annually. The median follow-up period for the assessment of OS was 3.4 years (range, 0.6-6.0 years) and for DFS was 3.1 years (range, 0.1-6.0 years). The study protocol was approved by the Ethics Committee of Osaka City University (\#926). Written informed consent was obtained from all subjects.

\section{Blood Sample Analysis}

Peripheral blood was obtained at the time of diagnosis, before NAC. The numbers of white blood cells were determined using a hemocytometer. The percentages of different types of cells were determined using a Coulter LH 750 Hematology Analyzer (Beckman Coulter, Brea, CA, USA). NLR was calculated from the preoperative blood sample by dividing the absolute neutrophil count by the absolute lymphocyte count. On the basis of previous studies, an NLR value of 3.0 was used as the cutoff value to discriminate between high-NLR $(\geq 3.0)$ and low-NLR $(<3.0)$. $^{18,29-31}$

\section{Statistical Analysis}

Statistical analysis was performed using the SPSS version 19.0 statistical software package (IBM, Armonk, NY, USA). We examined the associations between NLR and clinicopathologic variables using $\chi^{2}$ tests. Multivariate analysis of pCR was carried out using a binary logistic regression model. The Kaplan-Meier method was used to estimate DFS and OS, and the results between groups were compared using log-rank tests. Multivariate analysis of prognostic factors was carried out using a Cox regression model. A $p$ value $<0.05$ was considered significant. Cutoff values for different biomarkers included in this study were chosen before statistical analysis. ${ }^{18,29-32}$

\section{RESULTS}

\section{Clinicopathological Responses of Primary Breast}

Cancers to NAC

Clinical responses $(\mathrm{pCR}+$ partial response) were observed in 151 patients $(85.4 \%)$. NAC-related pCR was observed in 67 patients $(37.9 \%)$. The pCR rates were 
TABLE 1 Correlation between clinicopathological features and neutrophil to lymphocyte ratio in 177 all breast cancers

\begin{tabular}{|c|c|c|c|}
\hline \multirow[t]{2}{*}{ Parameters } & \multicolumn{2}{|l|}{$\operatorname{NLR}(n=177)$} & \multirow[t]{2}{*}{ ( $p$ value } \\
\hline & High $(n=119)$ & Low $(n=58)$ & \\
\hline Age at operation & & & 0.038 \\
\hline$\leq 56$ & $52(43.7 \%)$ & $35(60.3 \%)$ & \\
\hline$>56$ & $67(56.3 \%)$ & $23(39.7 \%)$ & \\
\hline Menopause & & & 0.037 \\
\hline Negative & $42(35.3 \%)$ & $30(51.7 \%)$ & \\
\hline Positive & $77(64.7 \%)$ & $28(48.3 \%)$ & \\
\hline Tumor size & & & 0.053 \\
\hline$\leq 2 \mathrm{~cm}$ & $20(16.8 \%)$ & $4(6.9 \%)$ & \\
\hline$>2 \mathrm{~cm}$ & $99(83.2 \%)$ & $54(93.1 \%)$ & \\
\hline Lymph node status & & & 0.552 \\
\hline Negative & $26(21.8 \%)$ & $15(25.9 \%)$ & \\
\hline Positive & $93(78.2 \%)$ & $43(74.1 \%)$ & \\
\hline Nuclear grade & & & 0.469 \\
\hline 1,2 & $94(79.0 \%)$ & $43(74.1 \%)$ & \\
\hline 3 & $25(21.0 \%)$ & $15(25.9 \%)$ & \\
\hline Ki67 & & & 0.292 \\
\hline$\leq 14 \%$ & $53(44.5 \%)$ & $21(36.2 \%)$ & \\
\hline$>14 \%$ & $66(55.5 \%)$ & $37(63.8 \%)$ & \\
\hline Intrinsic subtype & & & $<0.001$ \\
\hline TNBC & $25(21.0 \%)$ & $36(62.1 \%)$ & \\
\hline non-TNBC & $94(79.0 \%)$ & $22(37.9 \%)$ & \\
\hline Pathological & & & $<0.001$ \\
\hline $\mathrm{pCR}$ & $34(28.6 \%)$ & $33(56.9 \%)$ & \\
\hline non-pCR & $85(71.4 \%)$ & $25(43.1 \%)$ & \\
\hline
\end{tabular}

$N L R$ neutrophil-to-lymphocyte ratio, $T N B C$ triple-negative breast cancers, $p C R$ pathological complete response

$45.9 \%(28 / 61)$ and $33.6 \%(39 / 116)$ in patients with TNBC and with non-TNBC, respectively (Supplemental Table 1).

Among all cases, patients with pCR tended to have more favorable DFS ( $p=0.254$, log-rank) and OS $(p=0.221$, log-rank) compared with those with non-pCR, though the differences were not significant (Supplemental Fig. 1A, B). However, TNBC patients with pCR had significantly better DFS ( $p=0.043, \log$-rank) and OS $(p=0.049, \log$-rank $)$ than those with non-pCR (Supplemental Fig. 1C, D). There was no significant difference in DFS ( $p=0.964, \log$-rank) or OS ( $p=0.975, \log$-rank) in relation to $\mathrm{pCR}$ among patients with non-TNBC (Supplemental Fig. 1E, F).

\section{Associations Between Clinicopathological Parameters and NLR}

NLR was determined in every sample and ranged from 0.5 to 10.6 (mean, 2.3; median, 2.0; standard deviation 0.5 ). Fifty-eight patients were judged as having low NLR

TABLE 2 Correlations between neutrophil to lymphocyte ratio and clinicopathological parameters in 61 triple-negative breast cancers

\begin{tabular}{lll}
\hline Parameters & TNBC $(n=61)$ & ( $p$ value $)$ \\
\cline { 2 - 3 }$(n=25) \quad$ Low $(n=36)$ &
\end{tabular}

Age at operation

0.069

$\leq 56$

$>56$

Menopause

Negative

Positive

Tumor size

$\leq 2 \mathrm{~cm}$

$>2 \mathrm{~cm}$

Lymph node status

Negative

Positive

Nuclear grade

$$
1,2
$$

3

\section{Ki67}

\section{$\leq 14 \%$}

$>14 \%$

Pathological response

pCR

non-pCR

$\begin{array}{rr}8(32.0 \%) & 20(55.6 \%) \\ 17(68.0 \%) & 16(44.4 \%)\end{array}$

$7(28.0 \%) \quad 15(41.7 \%)$

$18(72.0 \%) \quad 21(58.3 \%)$

0.610

$3(12.0 \%) \quad 4(11.1 \%)$

$22(88.0 \%) \quad 32(88.9 \%)$

0.084

$$
2(8.0 \%) \quad 9(25.0 \%)
$$$$
23(92.0 \%) \quad 27(75.0 \%)
$$

$20(80.0 \%) \quad 24(66.7 \%)$

$5(20.0 \%) \quad 12(33.3 \%)$

0.002

$13(52.0 \%) \quad 5(13.9 \%)$

$12(48.0 \%) \quad 31(86.1 \%)$

$<0.001$

$N L R$ neutrophil-to-lymphocyte ratio, $T N B C$ triple-negative breast cancers, $p C R$ pathological complete response

(32.8\%) and 119 as high NLR (67.2\%). Low NLR was significantly correlated with younger age $(p=0.038)$, premenopausal status $(p=0.037)$, pCR result $(p<0.001)$, and TNBC phenotype $(p<0.001)$ (Table 1). Clinicopathological features were further investigated in TNBC patients. TNBC patients with low NLRs had high Ki67 indexes $(p=0.002)$ and were significantly more likely to achieve $\mathrm{pCR}(p<0.001)$. There was no significant correlation between NLR and any other tested clinicopathological parameters among TNBC patients (Table 2).

\section{Correlation Between NLR and Prognosis After NAC}

There was no significant difference in DFS or OS among all 177 patients (Figs. 1a, 2a) or among the 61 TNBC patients (Figs. 1b, 2b) stratified by NLR. However, among TNBC patients who achieved pCR, DFS ( $p=0.006$, logrank) and OS ( $p<0.001, \log$-rank) were both significantly longer in patients with low NLR compared with patients with high NLR (Figs. 1c, 2c). There was no significant difference in DFS or OS in patients with non-pCR in relation to NLR (Supplemental Fig. 2A-F). On univariate 
$\mathbf{A}$

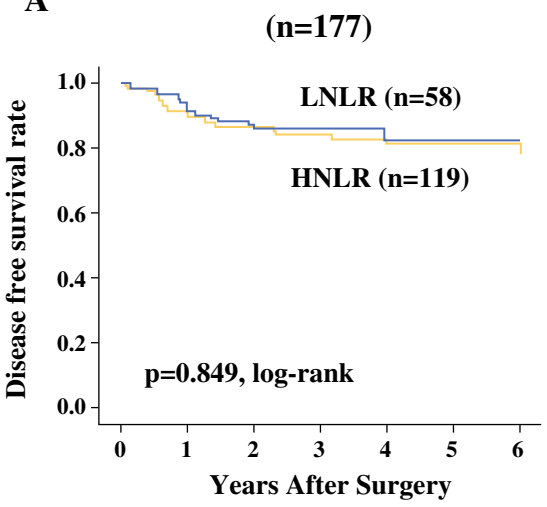

B

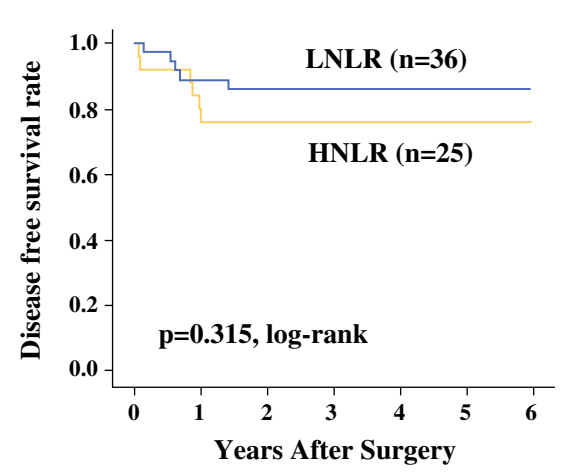

C

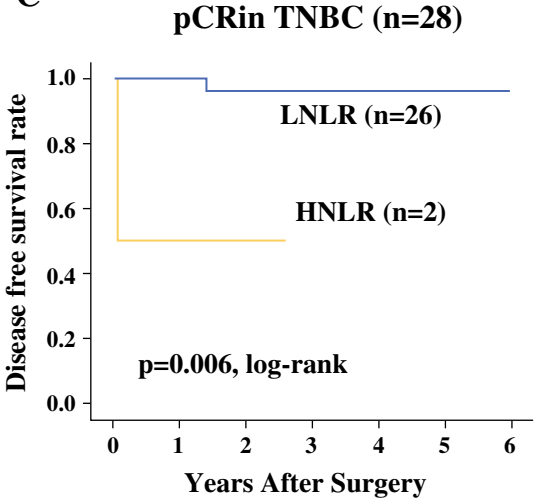

FIG. 1 There was no significant difference in disease-free survival (DFS) in relation to NLR among all 177 breast cancer patients (a), or among 61 TNBC patients (b). However, among TNBC patients who achieved pCR, DFS ( $p=0.006)$ was significantly longer in patients with low NLR after NAC, compared with patients with high NLR (c)

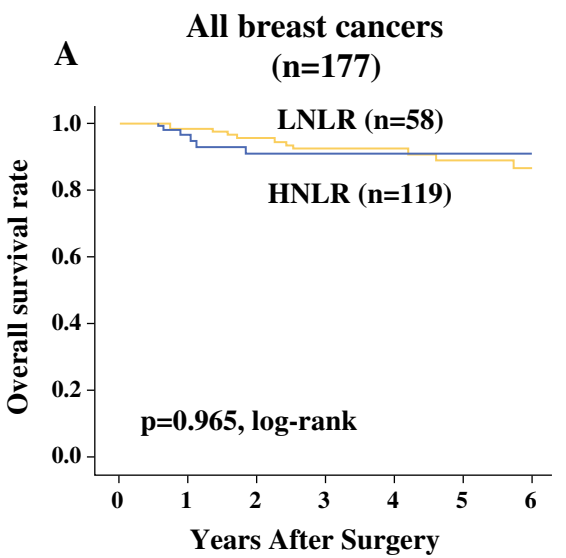

FIG. 2 There was no significant difference in overall survival (OS) in relation to NLR among all 177 breast cancer patients (a), or among 61 TNBC patients (b). However, among TNBC patients who achieved

analysis for recurrence, low NLR showed more favorable prognosis than high NLR $(p=0.044$, hazard ratio $(\mathrm{HR})=0.06)($ Table 3$)$. However, multivariate analysis also demonstrated that low NLR status was not an independent factor to indicate significantly more favorable prognosis of the patients compared with high-NLR status $(p=0.173, \mathrm{HR}=0.09)$.

\section{DISCUSSION}

NLR scores in breast cancer patients have been reported previously. ${ }^{18,19}$ However, the current study specifically enrolled patients who were eligible for NAC. Our results confirmed the correlation between high NLR and older age or post-menopausal status, as reported previously, ${ }^{18,19}$ suggesting that NLR may be influenced by the patient's systemic condition. We also found that patients with non-TNBC subtype had significantly higher
TNBC $(\mathbf{n}=61)$

LNLR (n=36)

HNLR $(n=25)$

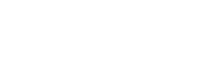
with non-TNBC was smaller $(116 / 177,65 \%)$ than in previous reports. ${ }^{18,19}$ In addition, the characteristics of the patients with non-TNBC were biased because of the exclusion of patients unsuitable for NAC, such as older patients and those with early-stage or disseminated disease. The current study population was therefore not suitable for investigating the correlation between NLR and tumor subtype.

Azab et al. studied 465 patients and demonstrated significantly poorer survival in those with high (highest quartile) NLR. ${ }^{19}$ Dircan et al. reported similar findings. ${ }^{33}$ Several other studies have also shown a correlation between high NLR and prognosis in breast cancer patients with selected features, such as luminal A phenotype. ${ }^{18}$ NLR is known to be particularly influenced in patients with advanced-stage disease, ${ }^{17,18}$ though patients with stage I or IV disease were eliminated from the current study. These 
TABLE 3 Univariable- and multivariable analysis with respect to disease-free survival in 61 triple-negative breast cancers

\begin{tabular}{|c|c|c|c|c|c|c|}
\hline \multirow[t]{2}{*}{ Parameter } & \multicolumn{3}{|c|}{ Univariable analysis } & \multicolumn{3}{|c|}{ Multivariable analysis } \\
\hline & Hazard ratio & $95 \%$ с.i. & $p$ value & Hazard ratio & $95 \%$ c.i. & $p$ value \\
\hline \multicolumn{7}{|l|}{ Lymph node status } \\
\hline Negative versus positive & 0.26 & $0.16-4.08$ & 0.334 & 0.45 & $0.14-14.44$ & 0.651 \\
\hline \multicolumn{7}{|l|}{ Ki67 (\%) } \\
\hline$\leq 14$ versus $>14$ & 0.74 & $0.22-2.53$ & 0.630 & 0.83 & $0.22-3.09$ & 0.781 \\
\hline \multicolumn{7}{|l|}{ NLR } \\
\hline Low versus high & 0.06 & $0.00-0.92$ & 0.044 & 0.09 & $0.00-2.89$ & 0.173 \\
\hline
\end{tabular}

$N L R$ platelet-to-lymphocyte ratio, c.i. confidence interval

strict inclusion criteria may explain the apparent lack of a clear correlation between NLR and prognosis.

The main factor influencing prognosis in our series was the efficacy of NAC, which was significantly correlated with NLR. Patients with a low NLR had a significantly higher pCR rate compared with patients with a high NLR. Moreover, the relationship between NLR and the efficacy of NAC differed between subtypes; NLR was significantly associated with NLR in patients with TNBC, but not in those with non-TNBC. The meaning of pCR is known to differ according to breast cancer subtype, and significant survival benefit only occurs in TNBC patients who achieve pCR. ${ }^{34}$ Effective biomarkers for predicting the efficacy of NAC in TNBC patient are therefore needed. In the present study, low NLR showed a close correlation with a favorable prognosis in patients with TNBC who achieved pCR. This observation may indicate the value of measuring NLR in TNBC patients who require NAC in order to predict the efficacy of the treatment.

In TNBC, the proliferation marker Ki67 has been suggested as the pCR predictive biomarker of NAC. ${ }^{35}$ And the TNBC patients in the present study showed a correlation between low NLR and the high Ki67 index group. Therefore, we thought that patients with a low NLR achieved a high pCR rate. However, in the factor analysis, only low NLR was useful as a favorable prognosis factor in NAC.

Breast cancer is not generally regarded as an immunerelated disorder. However, tumor-infiltrating lymphocytes and the expression of immune markers such as PD1, PDL1, and CTLA4 have recently been found to correlate with pCR in TNBC. ${ }^{36-41}$ NLR is relatively low in tumor subtypes with high lymphocyte activity, such as TNBC. ${ }^{42-44}$ Chemotherapy further activates the immune response in patients with low NLR, thereby accelerating the anti-tumor effect. $^{17-19,21,45}$

Recent studies have suggested that TNBC can be classified into seven subtypes, depending on the gene expression profile, ${ }^{44,46}$ and sensitivities to treatment are thought to differ among the different subtypes. ${ }^{34} \mathrm{BL}-1$ is a basal-like subtypes that demonstrates a high cell mass in culture along with high expression levels of cell cycle and DNA-injury-responsive genes, and which shows high chemosensitivity. In contrast, the BL-2 basal-like subtype and luminal androgen receptor subtype, which overexpresses androgen receptors, both have low pCR rates. ${ }^{34}$ The immunomodulatory subtype overexpresses genes related to immune reactions and has demonstrated higher sensitivity to chemotherapy and a more favorable prognosis compared with other subtypes. ${ }^{47}$ The results of the present study suggest that NLR may be correlated with TNBC subtypes and different outcomes after successful NAC.

In the present study, NLR was closely correlated with prognosis in TNBC patients after successful NAC, suggesting that low NLR may represent a useful surrogate marker in patients with TNBC. NLR can be measured easily without the need for any special equipment or invasive procedures. Further prospective studies are therefore warranted to confirm these preliminary results and to investigate the correlations between TNBC characteristics or subtypes and NLR.

ACKNOWLEDGMENT This study was supported in part by Grants-in Aid for Scientific Research (KAKENHI, Nos. 25461992 and 26461957) from the Ministry of Education, Culture, Sports, Science and Technology of Japan.

DISCLOSURE All of authors have no conflicts of interest to disclose.

OPEN ACCESS This article is distributed under the terms of the Creative Commons Attribution 4.0 International License (http:// creativecommons.org/licenses/by/4.0/), which permits unrestricted use, distribution, and reproduction in any medium, provided you give appropriate credit to the original author(s) and the source, provide a link to the Creative Commons license, and indicate if changes were made.

\section{REFERENCES}

1. Wolmark N, Wang J, Mamounas E, Bryant J, Fisher B. Preoperative chemotherapy in patients with operable breast cancer: nine-year results from National Surgical Adjuvant Breast and Bowel Project B-18. J Natl Cancer Inst Monogr. 2001(30):96102. 
2. van der Hage JA, van de Velde CJ, Julien JP, Tubiana-Hulin M, Vandervelden C, Duchateau L. Preoperative chemotherapy in primary operable breast cancer: results from the European Organization for Research and Treatment of Cancer trial 10902. $J$ Clin Oncol. 2001;19(22):4224-37.

3. Mayer EL, Carey LA, Burstein HJ. Clinical trial update: implications and management of residual disease after neoadjuvant therapy for breast cancer. Breast Cancer Res. 2007;9(5):110.

4. Sachelarie I, Grossbard ML, Chadha M, Feldman S, Ghesani M, Blum RH. Primary systemic therapy of breast cancer. Oncologist. 11(6):574-89.

5. Bear HD, Anderson S, Brown A, et al. The effect on tumor response of adding sequential preoperative docetaxel to preoperative doxorubicin and cyclophosphamide: preliminary results from National Surgical Adjuvant Breast and Bowel Project Protocol B-27. J Clin Oncol. 2003;21(22):4165-74.

6. Henderson IC, Berry DA, Demetri GD, et al. Improved outcomes from adding sequential Paclitaxel but not from escalating Doxorubicin dose in an adjuvant chemotherapy regimen for patients with node-positive primary breast cancer. J Clin Oncol. 2003;21(6):976-83.

7. Valachis A, Mauri D, Polyzos NP, Chlouverakis G, Mavroudis D, Georgoulias V. Trastuzumab combined to neoadjuvant chemotherapy in patients with HER2-positive breast cancer: a systematic review and meta-analysis. Breast. 2011;20(6):485-90.

8. Gianni L, Eiermann W, Semiglazov V, et al. Neoadjuvant chemotherapy with trastuzumab followed by adjuvant trastuzumab versus neoadjuvant chemotherapy alone, in patients with HER2-positive locally advanced breast cancer (the NOAH trial): a randomised controlled superiority trial with a parallel HER2negative cohort. Lancet. 2010;375(9712):377-84.

9. Abd El-Rehim DM, Ball G, Pinder SE, et al. High-throughput protein expression analysis using tissue microarray technology of a large well-characterised series identifies biologically distinct classes of breast cancer confirming recent cDNA expression analyses. Int J Cancer. 2005;116(3):340-50.

10. Mattie MD, Benz CC, Bowers J, et al. Optimized high-throughput microRNA expression profiling provides novel biomarker assessment of clinical prostate and breast cancer biopsies. Mol Cancer. 2006;5:24.

11. Perou CM, Sorlie T, Eisen MB, et al. Molecular portraits of human breast tumours. Nature. 2000;406(6797):747-52.

12. Bauer KR, Brown M, Cress RD, Parise CA, Caggiano V. Descriptive analysis of estrogen receptor (ER)-negative, progesterone receptor (PR)-negative, and HER2-negative invasive breast cancer, the so-called triple-negative phenotype: a population-based study from the California cancer Registry. Cancer. 2007;109(9):1721-28

13. Nielsen TO, Hsu FD, Jensen K, et al. Immunohistochemical and clinical characterization of the basal-like subtype of invasive breast carcinoma. Clin Cancer Res. 2004;10(16):5367-74.

14. Rakha EA, El-Rehim DA, Paish C, et al. Basal phenotype identifies a poor prognostic subgroup of breast cancer of clinical importance. Eur J Cancer. 42(18):3149-56.

15. Sorlie T, Perou CM, Tibshirani R, et al. Gene expression patterns of breast carcinomas distinguish tumor subclasses with clinical implications. Proc Natl Acad Sci USA. 2001;98(19): 10869-74.

16. Sorlie T, Tibshirani R, Parker J, et al. Repeated observation of breast tumor subtypes in independent gene expression data sets. Proc Natl Acad Sci USA. 2003;100(14):8418-23.

17. Azab B, Shah N, Radbel J, et al. Pretreatment neutrophil/lymphocyte ratio is superior to platelet/lymphocyte ratio as a predictor of long-term mortality in breast cancer patients. Med Oncol. 2013;30(1):432.
18. Noh H, Eomm M, Han A. Usefulness of pretreatment neutrophil to lymphocyte ratio in predicting disease-specific survival in breast cancer patients. J Breast Cancer. 2013;16(1):55-9.

19. Azab B, Bhatt VR, Phookan J, et al. Usefulness of the neutrophil-to-lymphocyte ratio in predicting short- and long-term mortality in breast cancer patients. Ann Surg Oncol. 2012;19(1):217-24.

20. Malietzis G, Giacometti M, Kennedy RH, Athanasiou T, Aziz O, Jenkins JT. The emerging role of neutrophil to lymphocyte ratio in determining colorectal cancer treatment outcomes: a systematic review and meta-analysis. Ann Surg Oncol. 2014;21(12): 3938-46.

21. Templeton AJ, McNamara MG, Seruga B, et al. Prognostic role of neutrophil-to-lymphocyte ratio in solid tumors: a systematic review and meta-analysis. J Natl Cancer Inst. 2014;106(6): dju124.

22. Rossi L, Santoni M, Crabb SJ, et al. High neutrophil-to-lymphocyte ratio persistent during first-line chemotherapy predicts poor clinical outcome in patients with advanced urothelial cancer. Ann Surg Oncol. Sep 192014.

23. Luo G, Guo M, Liu Z, et al. Blood Neutrophil-Lymphocyte Ratio Predicts Survival in Patients with Advanced Pancreatic Cancer Treated with Chemotherapy. Ann Surg Oncol. Aug 262014.

24. Singletary SE, Greene FL, Sobin LH. Classification of isolated tumor cells: clarification of the 6th edition of the American Joint Committee on Cancer Staging Manual. Cancer. 2003;98(12): 2740-41.

25. Mauri D, Pavlidis N, Ioannidis JP. Neoadjuvant versus adjuvant systemic treatment in breast cancer: a meta-analysis. J Natl Cancer Inst. 2005;97(3):188-94.

26. Mieog JS, van der Hage JA, van de Velde CJ. Preoperative chemotherapy for women with operable breast cancer. Cochrane Database Syst Rev. 2007(2):CD005002.

27. Buzdar AU, Valero V, Ibrahim NK, et al. Neoadjuvant therapy with paclitaxel followed by 5-fluorouracil, epirubicin, and cyclophosphamide chemotherapy and concurrent trastuzumab in human epidermal growth factor receptor 2-positive operable breast cancer: an update of the initial randomized study population and data of additional patients treated with the same regimen. Clin Cancer Res. 2007;13(1):228-33.

28. Eisenhauer EA, Therasse P, Bogaerts J, et al. New response evaluation criteria in solid tumours: revised RECIST guideline (version 1.1). Eur J Cancer. 2009;45(2):228-47.

29. Perez DR, Baser RE, Cavnar MJ, et al. Blood neutrophil-tolymphocyte ratio is prognostic in gastrointestinal stromal tumor. Ann Surg Oncol. 2013;20(2):593-99.

30. Yamanaka T, Matsumoto S, Teramukai S, Ishiwata R, Nagai Y, Fukushima M. The baseline ratio of neutrophils to lymphocytes is associated with patient prognosis in advanced gastric cancer. Oncology. 2007;73(3-4):215-20.

31. Dutta S, Crumley AB, Fullarton GM, Horgan PG, McMillan DC. Comparison of the prognostic value of tumour- and patient-related factors in patients undergoing potentially curative resection of oesophageal cancer. World J Surg. 2011;35(8):1861-66.

32. Inoue D, Ozaka M, Matsuyama M, et al. Prognostic value of neutrophil-lymphocyte ratio and level of C-reactive protein in a large cohort of pancreatic cancer patients: a retrospective study in a single institute in Japan. Jpn J Clin Oncol. Oct 232014.

33. Dirican A, Kucukzeybek BB, Alacacioglu A, et al. Do the derived neutrophil to lymphocyte ratio and the neutrophil to lymphocyte ratio predict prognosis in breast cancer? Int J Clin Oncol. Feb 182014.

34. Masuda H, Baggerly KA, Wang Y, et al. Differential response to neoadjuvant chemotherapy among 7 triple-negative breast cancer molecular subtypes. Clin Cancer Res. 2013;19(19):5533-40. 
35. Denkert C, Loibl S, Muller BM, et al. Ki67 levels as predictive and prognostic parameters in pretherapeutic breast cancer core biopsies: a translational investigation in the neoadjuvant GeparTrio trial. Ann Oncol. 2013;24(11):2786-93.

36. Adams S, Gray RJ, Demaria S, et al. Prognostic Value of TumorInfiltrating Lymphocytes in Triple-Negative Breast Cancers From Two Phase III Randomized Adjuvant Breast Cancer Trials: ECOG 2197 and ECOG 1199. J Clin Oncol. Jul 282014.

37. Dieci MV, Criscitiello C, Goubar A, et al. Prognostic value of tumor-infiltrating lymphocytes on residual disease after primary chemotherapy for triple-negative breast cancer: a retrospective multicenter study. Ann Oncol. 2014;25(3):611-8.

38. Loi S, Michiels S, Salgado R, et al. Tumor infiltrating lymphocytes are prognostic in triple negative breast cancer and predictive for trastuzumab benefit in early breast cancer: results from the FinHER trial. Ann Oncol. 2014;25(8):1544-50.

39. Loi S, Sirtaine N, Piette F, et al. Prognostic and predictive value of tumor-infiltrating lymphocytes in a phase III randomized adjuvant breast cancer trial in node-positive breast cancer comparing the addition of docetaxel to doxorubicin with doxorubicin-based chemotherapy: BIG 02-98. J Clin Oncol. 2013;31(7):860-7.

40. Schalper KA, Velcheti V, Carvajal D, et al. In situ tumor PD-L1 mRNA expression is associated with increased TILs and better outcome in breast carcinomas. Clin Cancer Res. 2014;20(10): 2773-82.
41. Velcheti V, Schalper KA, Carvajal DE, et al. Programmed death ligand-1 expression in non-small cell lung cancer. Lab Invest. 2014;94(1):107-16.

42. Desmedt C, Haibe-Kains B, Wirapati P, et al. Biological processes associated with breast cancer clinical outcome depend on the molecular subtypes. Clin Cancer Res. 2008;14(16):5158-65.

43. Kreike B, van Kouwenhove M, Horlings H, et al. Gene expression profiling and histopathological characterization of triplenegative/basal-like breast carcinomas. Breast Cancer Res. 2007; 9(5):R65

44. Lehmann BD, Bauer JA, Chen X, et al. Identification of human triple-negative breast cancer subtypes and preclinical models for selection of targeted therapies. J Clin Invest. 2011;121(7):275067.

45. Mahmoud SM, Paish EC, Powe DG, et al. Tumor-infiltrating CD8 + lymphocytes predict clinical outcome in breast cancer. J Clin Oncol. 2011;29(15):1949-55.

46. Metzger-Filho O, Tutt A, de Azambuja E, et al. Dissecting the heterogeneity of triple-negative breast cancer. J Clin Oncol. 2012;30(15):1879-87.

47. Andre F, Dieci MV, Dubsky P, et al. Molecular pathways: involvement of immune pathways in the therapeutic response and outcome in breast cancer. Clin Cancer Res. 2013;19(1):28-33. 